\title{
On the possibility of the long-term forecast of solar activity using radiocarbon sunspot proxy
}

\author{
M.G. Ogurtsov ${ }^{1}$ \\ ${ }^{1}$ Ioffe Physico-Technical Institute, Russian Academy of Sciences, Politekhnicheskaya str. 26, \\ 194021 St.Petersburg, Russia e-mail: Maxim.Ogurtsov@mail.ioffe.ru
}

\begin{abstract}
The reconstruction of sunspot numbers from the decadal radiocarbon series was made for the time interval 8005 B.C. - 1945 A.D using five-box carbon exchange model. Analyses showed apparent persistency in the reconstructed Wolf number series. It indicates the existence of long-term memory in solar activity. Nonlinear forecast of the mean value of Wolf number is made for the next 40 years using obtained radiocarbon proxy. Comparison of the results with the long -term forecasts of the other authors was performed.
\end{abstract}

\section{Introduction}

Radiocarbon is well known solar proxy. But carbon exchange system works as a lowpass filter, and, therefore, can significantly distort statistical properties of solar activity. This shortcoming can be diminished if use a reconstructed sunspot number instead of raw $\Delta^{14} C$ data. In present work Wolf numbers, reconstructed from decadal radiocarbon series of Stuiver et al. (1998) using five-reservoir model of carbon exchange system (see Ogurtsov (2004)for details), were analyzed. The reconstructed series (here and after CW) is plotted in figure $1^{a}$.

\section{Results and discussion}

Rescale analysis and detrended fluctuation analysis were used for investigation of memory effects of the data set CW. These analyses are based on investigation of scaling properties of statistical values $R / S$ and DFA, which structure is connected with cumulative time series. As seen from figure $1^{b, c}$ scaling exponent of both statistics is larger than 0.5 that testifies presence of long-term memory.

Presence of long-term memory should mean possibility of long-range (time more than 11 years) sunspot forecast. This prognosis was made using nonlinear forecast technique. This approach is based on investigation of evolution of system's trajectory in multidimensional pseudo-phase space. Results of forecast of sunspot activity for the first part of XXI century, obtained by means of original program and by programs of TISEAN package (Kantz et al. (1999))are shown in figure 2 (d - embedding dimension, $\tau$ - time delay). Predictions of other authors also are plotted in the figure. It is seen from figure 2 that despite appreciable differences almost all forecasts have a common feature - they indicate mean level of sunspot activity in the first part of XXI century lower than that in the second part of XX century. Thus one can conclude, that more likely solar activity will decline during the next 40-50 years.

\section{Acknowledgements}

This research was done in the frame of an exchange between the Russian and Finnish Academies (project 16) and was supported by EU INTAS 2001-0550 grant. In addition, 


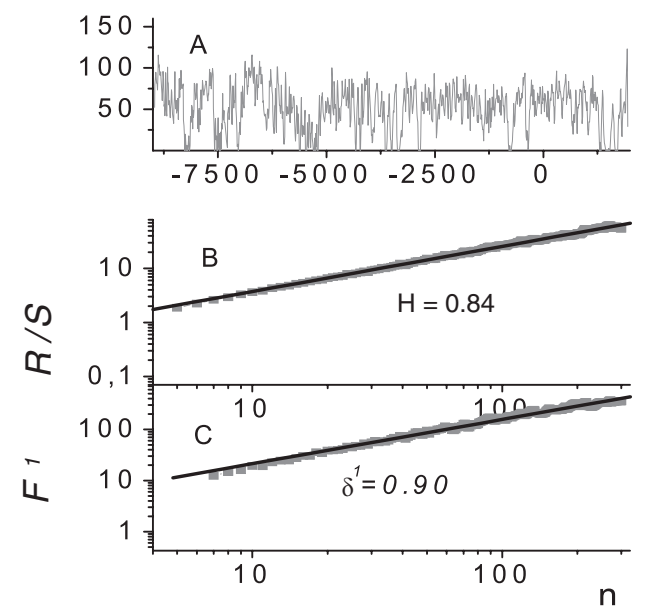

Figure 1. A - CW time series -Wolf numbers, reconstructed from $\Delta^{14} C$ data of Stuiver et al. (1998); B, C - the quantities $R / S$ and $F^{1}$ as function of scale $n$ calculated for CW

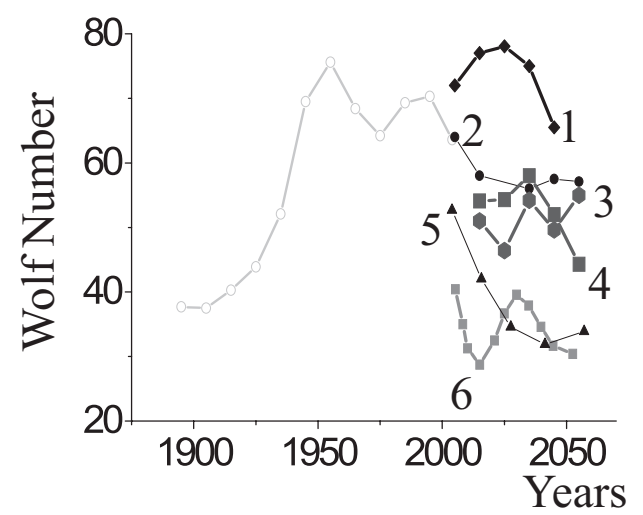

Figure 2. Actual Wolf number smoothed by 25 years (gray line with opened circles); forecast of sunspot number made in present work using 4 nearest neighbors, $d=3, \tau=1$ (line 4 ) and 5 nearest neighbors, $d=4, \tau=1$ (line 3 ); forecast of sunspot number made in present work using TISEAN program nstep with $d=3, \tau=1$ (line 2); forecasts of sunspot number made by Nagovitsyn and Ogurtsov (2003, line 6), Miletskij (2003, line 5), Vasiliev et al. (2002, line 1)

it was supported by the program "Astronomy: nonstationary processes in astronomy" of Russian Academy and by RFBR grants 03-02-17505, 03-04-48769, 04-02-17560.

\section{References}

Miletskij E.V. 2003 In Proc. of VII Pulkovo Conference on solar physics, 7-11 June 2003, St.Petersburg, 305-313. (in Russian).

Nagovitsyn Yu.A.\& Ogurtsov M.G. 2003 In Proc. of VII Pulkovo Conference on solar physics, 7-11 June 2003, St.Petersburg, 327-332. (in Russian).

Ogurtsov M.G. 2004 Solar Physics 220, 93-105.

Stuiver M., Reimer P.J. \& Braziunas T.F. 1998 Radiocarbon 40, 1127-1151.

Vasiliev S.S., Dergachav V.A. \& Raspopov O.M. 2002 Geomagnetism and Aeronomy 42, 147154 (in Russian).

Hegger R., Kantz H. \& Schreiber T. 1999 CHAOS 9, 413-435. 